\title{
Oral Manifestations of a Patient Suffering from a Rare Gilbert's Syndrome: A Case Report
}

\author{
Hira Abbasi ${ }^{1}$, Abhishek Lal ${ }^{2}$, Rizwan Jouhar ${ }^{3}$ \\ ${ }^{1}$ FCPS Part II Trainee, Department of Operative Dentistry, Altamash Institute of Dental Medicine, Karachi \\ ${ }^{2}$ BDS, Department of Operative Dentistry, Altamash Institute of Dental Medicine, Karachi \\ ${ }^{3}$ Assistant Professor, Department of Restorative Dentistry and Endodontics, King Faisal University, Al-Ahsa, Saudi \\ Arabia
}

Gilbert's syndrome is a rare genetic disorder characterized by abnormal glucuronidation of bilirubin in the liver, presenting as unconjugated hyperbilirubinemia in the absence of hepatocellular injury or hemolysis. Diagnosis of this pathology is primarily made during routine examination described as the presence of a yellowish tinge in the eyes and skin in general. Normally, the oral manifestations of Gilbert's syndrome are present but mostly go unnoticed as the teeth are minimally affected which are visible to the patient and surrounding mucosa in the oral cavity, where yellow discoloration can be appreciated. Dental treatments are smoothly carried out for such patients like extractions, root canal treatment, cleaning prophylaxis. The patient in this case safely underwent the root canal treatment after being diagnosed with irreversible pulpitis without any unusual discomfort. Local anesthesia can also be safely administered to such patients such as infiltration and inferior alveolar block anesthesia.

Keywords: Gilberts Syndrome, Oral, Manifestations

\begin{tabular}{ll}
\hline Correspondence: & Article info: \\
Hira Abbasi & Received: April 19, 2021 \\
Email: drhirabbasi@gmail.com & Accepted: August 31, 2021 \\
\hline
\end{tabular}

Cite this case Report: Abasi H, Lal A, Jouhar R. Oral Manifestations of a Patient Suffering from a Rare Gilbert's Syndrome: A Case Report. J Islamabad Med Dental Coll. 2021; 10(3): 181-185.

Funding Source: Nil Doi: $10.35787 / j i m d c . v 10 i 3.706$

Conflict of Interest: Nil

\section{Introduction}

Gilbert's syndrome (GS) is a rare autosomal recessive genetic disorder characterized by abnormal metabolism (glucuronidation) of bilirubin in the liver, presenting as unconjugated hyperbilirubinemia in the absence of hepatocellular injury or hemolysis. ${ }^{1}$ Normally patients do not report any symptoms that might hinder their daily lives so this pathology is mostly diagnosed during a routine examination. Generally, it manifests as neonatal jaundice, abdominal pain, fatigue, loss of appetite, nausea, irritable bowel syndrome, and brain fog. Symptoms worsen when the bilirubin levels tend to increase above $7 \mathrm{mg} / \mathrm{dL}$.

About its prevalence, Gilbert's syndrome has been reported to vary amongst different populations between $4 \%-16 \% .^{2}$ Moreover, during adolescence, there is a change in steroids concentration which tends to affect the metabolism of bilirubin that then leads to increased bilirubin levels. Gilbert's syndrome further is most commonly found in males as compared to females, primarily due to the greater daily production of bilirubin in males. ${ }^{3}$

Although the pathology itself is harmless for the patient, symptoms that get the patient's attention tend to increase anxiety amongst them so psychological counselling becomes an important part of the treatment plan. So, the diagnostic criteria to achieve a definitive diagnosis of Gilbert's syndrome include an assessment of bilirubin levels as part of the Liver Function Test. Moreover, mildly 
elevated bilirubin levels alongside normal serum liver transaminases, red blood cell count, and biliary damage markers may indicate the presence of Gilbert's syndrome. Furthermore, mildly elevated unconjugated bilirubin in GS is associated with the presence of reduced prevalence of chronic diseases, like cardiovascular diseases particularly, and type 2 diabetes along with its risk factors. ${ }^{4}$

The most characteristic feature that can be clinically appreciated in such patients is the yellow tinge of the skin and the eyes particularly. ${ }^{5}$ Skin and mucosa of the body are affected to various degrees along with these manifestations found in the oral cavity. Very scarcely, studies report the prevalence of oral manifestations of patients suffering from GS, mainly due to its signs been overlooked in the oral cavity and more conspicuous signs being focused upon by the treating physician. Although the oral manifestations of GS if present are still harmless and a patient can undergo any dental treatment, the presence of such signs in the oral cavity should be searched for.

\section{Case Report}

A 35 years old female presented to the outpatient department of Operative Dentistry of Altamash Institute of Dental Medicine (Karachi, Pakistan). Earlier 1 month ago, the patient developed severe throbbing pain in the lower left side of the jaw, unable to point out the tooth from where the pain was originating. The pain was persistently present, not completely relieved by self-medication as reported in the chief complaint of the patient. Furthermore, the patient had difficulty in eating with the pain radiating to the neck and temporal regions of the head of the affected side that awakened her in the middle of the sleep and aggravated especially during sleeping hours. Previously before visiting the dentist for treatment, the patient reportedly took some painkillers to relieve the pain but the effect was mild and still being bothersome for the patient, mandating a visit to the Outpatient Department.

Regarding medical history, the patient had been diagnosed with Gilbert's syndrome a couple of years back for which she had not been prescribed any medicines. Other than that, the patient had no significant medical and family history, along with dental and social history. No allergies to drugs were reported by the patient. Previously, the patient had undergone dental treatment such as extractions which were recommended to her without any unfortunate events during the perioperative and postoperative phases, as per the patient's own words. The patient was diagnosed with irreversible pulpitis with the lower-left $1^{\text {st }}$ molar being the offending tooth, for which non-vital therapy i.e., a pulpectomy (Root canal Treatment) had been suggested by the doctor as confirmed by intraoral, extraoral, and radiological examinations, respectively.

As the patient reportedly suffered from Gilbert's Syndrome, upon intraoral examinations some remarkable features were noted. The patient had a deep palate with a yellow tinge mainly limited to the hard palate and fading away towards the soft palate as shown in figure 1. Furthermore, on examining the floor of the mouth, a similar yellow color was noted with the mucosa along sublingual folds, lingual frenum, and sublingual caruncle as shown in figure 2. Regarding the appearance of the tongue, the size of the tongue was unremarkable with a normal shape although a smooth appearance was noted around the tip of the tongue and anterolateral parts of the tongue as shown in figure 3. The lower lip when being everted for examination was found to have a yellowish appearance diffusely present all around as shown in figure 4. The patient had normal teeth with some missing due to caries and trauma with slight paleness around the free and attached gingiva generalized around all of the teeth, and generalized spacing too, as shown in figure 5 . While performing an extra-oral examination, the patient had normal 
temporomandibular joint movements, normal mouth opening, anatomy of the lips was unremarkable and no other remarkable features were found on the facial aspect of the patient. Lastly, the most obvious clinical finding in this patient was yellow discoloration of the eyes. No other remarkable features were noted in the rest of the body.

\section{Fig 1: Hard and Soft Palate}

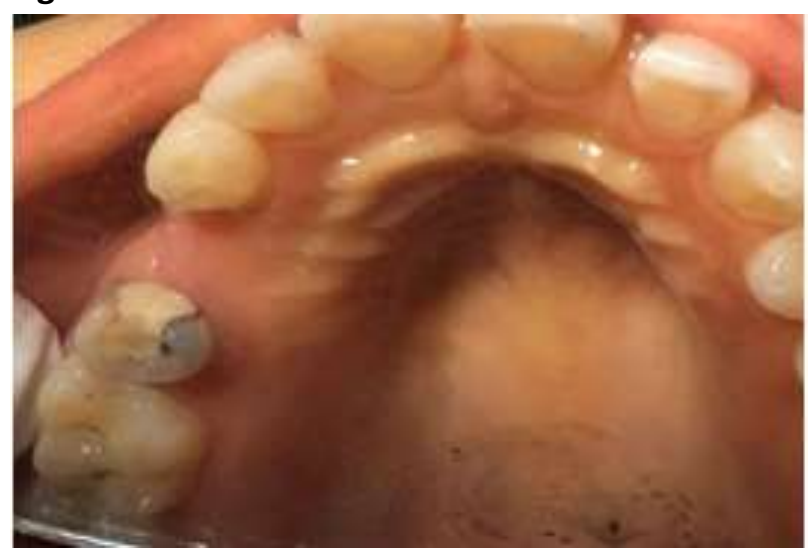

Fig 2: Floor of the Mouth

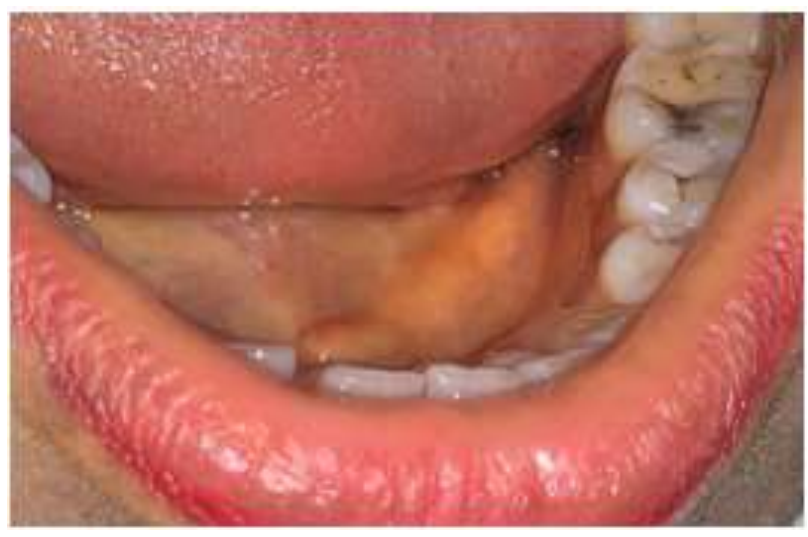

Fig 3: Tongue

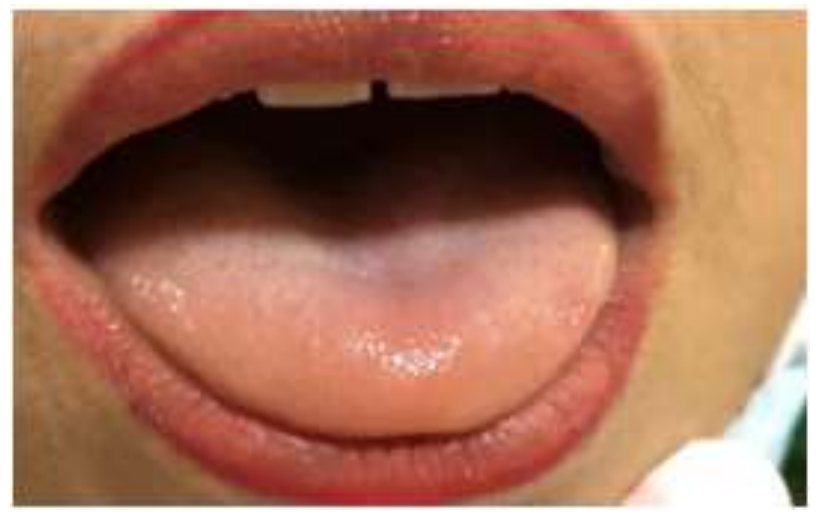

\section{Fig 4: Everted Lower Lip}

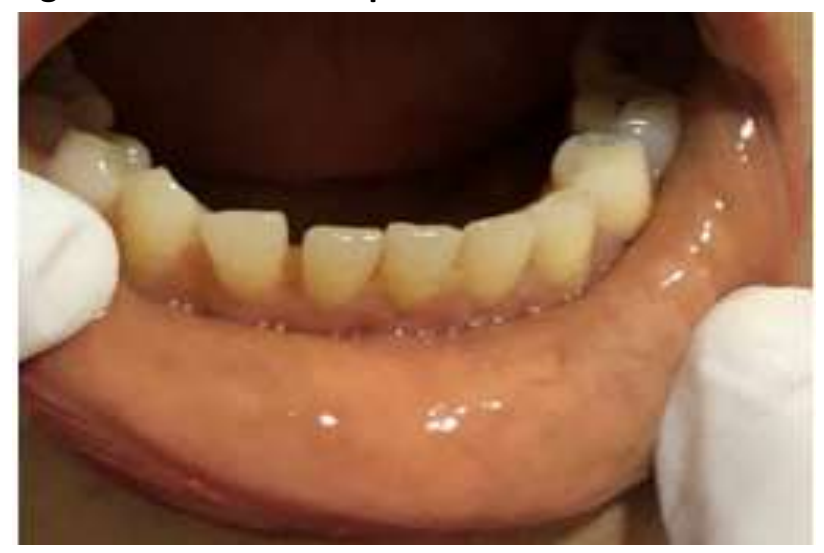

Fig 5: Teeth and Gingiva

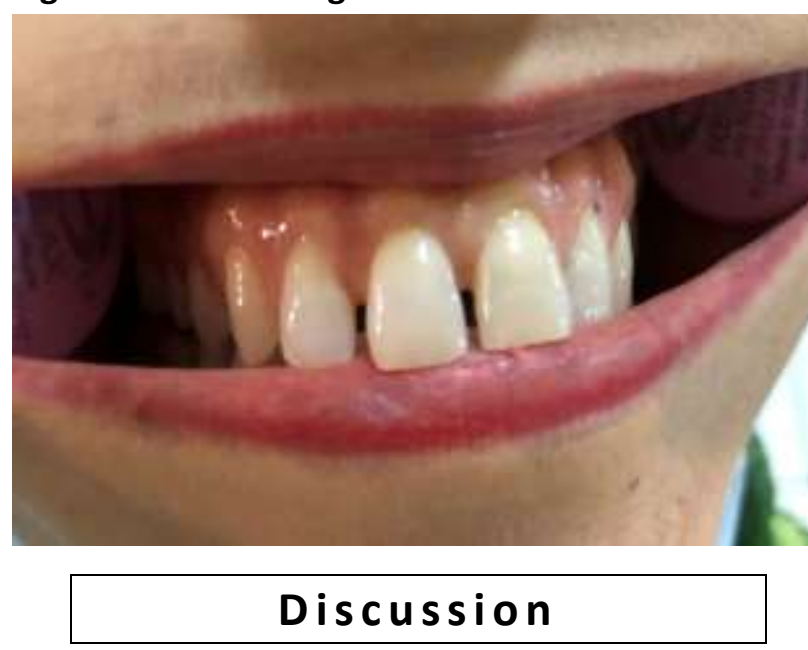

Gilbert's syndrome is a rare genetic disorder, which although alerts the patient when looked upon, but is harmless in general. Normally the patients do not report any disturbing signs and symptoms in general and in regards to the oral cavity. The clinical manifestations only become more noticeable in certain situations such as fatigue, exercise, febrile illness, fasting, and alcohol ingestion especially in older patients. ${ }^{6}$

In our case report, it was found that the patient showed a yellow tinge as oral manifestation in the lower lip, hard palate, the floor of the mouth, gingiva, and tongue. No abnormalities were noted regarding teeth such as number, shape, or size. Moreover, the patient also had yellow discoloration in the eyes and on the skin in general. Sometimes as the literature states, Crigler-Najjar Syndrome 
also manifests similarly to Gilbert's syndrome, so it's important to reach a definitive diagnosis correctly for the proper management of the patient. $^{7}$

Keeping in mind the oral manifestations of Gilberts syndrome, patients normally do not notice these signs and symptoms for consultation from a physician or a dentist. Brushing is the primary focus of the patients and since this pathology hasn't been reported to affect the teeth as such in the literature, other signs go unnoticed. Most commonly, it's the general body symptoms that alert the patients suspected of suffering from GS that make them visit the doctor.

In regards to the dental procedure, patients suffering from GS normally do not suffer from any discomfort in any of the dental procedures required although literature reports some patients did develop jaundice following oral surgery procedures. ${ }^{8}$ Moreover, as local anesthesia was used in the patient being treated in our Outpatient Department, no difficulties for the doctor as well as the patient were reported till the next visit of the patient which was 1 week later. However, studies report problems with regional and general anesthesia for patients suffering from GS so the administering agents should be carefully selected as the hepatic dysfunction is present and it is the liver where the drugs metabolize. ${ }^{9,10}$ The patient in our case safely went through the entire root canal treatment from chamber opening, pulpectomy, cleaning and shaping, obturation, core build-up, crown prep, and finally cementation. No unusual discomfort was reported by the patient other than the normal sensations felt during any dental procedure by every normal and healthy patient such as pressure sensations.

\section{Conclusion}

Although a rare syndrome and being harmless in general, Gilbert's syndrome until not being diagnosed does raise some anxiety in the sufferers.
So, assurance along with necessary treatments must be offered to such patients with dietary guidance too. Concerning the dental part of the syndrome, although yellow discoloration is present in the oral cavity, it's mostly harmless and patients safely undergo different dental treatments along with administration of local anesthesia with no reported side effects.

\section{References}

1. Kamal S, Abdelhakam S, Ghoraba D, Massoud Y, Aziz KA, Hassan H, Hafez T, Sallam AA. The frequency, clinical course, and health related quality of life in adults with Gilbert's syndrome: a longitudinal study. BMC gastroenterology. 2019; 19(1):1-4. Doi: 10.1186/s12876-019-0931-2

2. Zaman S, Fukushima $H$, Suzuki $R$, Hawlader MD, Yoshimatsu S, Kanai Y, Ahsan GU, Fukushima T. Prevalence of Gilbert syndrome in Bangladesh. Open Journal of Blood Diseases. 2018; 8(01):1. Doi: 10.4236/ojbd.2018.81001

3. Creeden JF, Gordon DM, Stec DE, Hinds Jr TD. Bilirubin as a metabolic hormone: the physiological relevance of low levels. Am. J. Physiol. Endocrinol. Metab. 2021; 320(2):E191-207. Doi: 10.1152/ajpendo.00405.2020

4. English E, Lenters-Westra E. HbA1c method performance: the great success story of global standardization. Crit Rev Clin Lab Sci. 2018; 55(6):408-19. Doi: 10.1080/10408363.2018.1480591

5. Qian JD, Hou FQ, Wang TL, Shao C, Wang GQ. Gilbert syndrome combined with prolonged jaundice caused by contrast agent: Case report. World J Gastroentero. 2018; 24(13):1486. Doi: 10.3748/wjg.v24.i13.1486

6. Tzoneva D, Aleksiev E, Michova K, Stanimirov P. Perioperative management and anaesthetic considerations for adult patients with Gilbert's syndrome and oral cancer: review and case report. Biotechnol Biotechnol Equip. 2019; 33(1):1182-6. doi: 10.1080/13102818.2019.1649987

7. Stanley LA. Drug metabolism. InPharmacognosy 2017: (pp. 527-545). Academic Press. Doi: 10.1016/B978-0-12-802104-0.00027-5

8. Quinn NW, Gollan JL. Jaundice following oral surgery: Gilbert's syndrome. Br J Oral Maxillofac Surg. 1975;12(3):285-8. Doi: 10.1016/0007117X(75)90059-1

9. Nag D, Sinha N, Samaddar D, Mahanty P. General anesthesia in a patient with Gilbert's syndrome. J Anaesthesiol Clin Pharmacol. 2011;27(2):253. Doi: 
10.4103/0970-9185.81836

10. Ranjan RV, Ramachandran TR, Veliath DG, Coelho D. Perioperative management of a patient with Gilberts syndrome and rheumatic heart disease.
Saudi J Anaesth.. 2012;6(3):289. Doi: 10.4103/1658354X.101225 